\section{The Spectrum of Iron.}

F. M. Walters, Jr. (Journ. Wash. Acad. Sci. I3, 1923, p. 243) has found twenty " multiplets" in the iron arc spectrum of the kind which were first found by M. A. Catalán in the spectrum of manganese (Phil. Trans. A, 223, I922, p. I27). He also gives the Zeeman patterns of some lines. The structure of such multiplets, which are combinations of multiple terms, is governed by the restriction rules for the " inner" quantum numbers $J$, given by Sommerfeld and Landé (Zeit. f. Phys. I5, p. I 89). With the help of these rules it is not difficult to find the inner quantum numbers of the terms, unknown for the rest, of which the given multiplets are combinations.

In most cases it is only possible to find relative values for these quantum numbers $J$, but in some cases, and fortunately in this one, there is one rule helping us to get the absolute values. This rule is the combination interdiction of two terms having both the inner quantum number $J=\frac{1}{2}$ (Landé, l.c.). It gives us the absolute values of $J$ for the terms of multiplets wherein an initially expected spectral line is missing. As in our case the same term differences, and thus the same terms, occur in several multiplets, it is easy to obtain also with considerable certainty the absolute values of $J$ for all the other terms. The Table 3 in the work of Landé gives us then for each multiple term the quantum numbers $k$ and $\mathrm{R}$; that means, we can find the names and the systems of the spectral terms. In some cases we need the indications of the Zeeman effect to decide between two possibilities. These Zeeman indications, moreover, give a very good confirmation for the results.

The results have shown that the twenty iron multiplets belong to a triplet and a quintuplet system. Two of the thirteen different terms are irregular; perhaps those concerning multiplets were not completely observed by Mr. Walters. The names of the terms with their quantum numbers and differences are as follows:

Triplet system :

$$
\begin{aligned}
& k=3 J=\frac{7}{2}, \frac{5}{2}, \frac{3}{2} d^{\prime} \text {, } \\
& k=4 J=\frac{7}{2}, \frac{7}{2}, \frac{5}{2}\left\{\begin{array}{l}
f^{\prime}, \\
f^{\prime \prime},
\end{array}\right. \\
& \Delta \nu=390 \cdot 6,252 \cdot 0 \\
& =584 \cdot 7,407 \cdot 6 \\
& =476 \cdot 5,358 \cdot 5 \\
& =388.4,3 \mathrm{II} \cdot 8
\end{aligned}
$$

Quintuplet system :

$$
\begin{aligned}
& J=\frac{g}{2}, \frac{7}{2}, \frac{5}{2}, \frac{3}{2}, \frac{1}{2}\left\{\begin{array}{l}
D^{\prime}, \\
D^{\prime \prime} \\
D^{\prime \prime \prime}, \\
D^{\prime \prime},
\end{array}\right. \\
& \Delta \nu=4 \mathrm{I} 5 \cdot 9,288 \cdot \mathrm{O}, \mathrm{I} 84^{\cdot} \mathrm{I}, \\
& =344^{\circ} 0,26 I \cdot 5, I 73 \cdot 2 \text {, } \\
& =240 \cdot 2 \text {, I99.5, I39.7, } 7 \mathrm{I} \cdot \mathrm{I} \\
& =384 \cdot 3,272 \cdot 6, I 75 \cdot 2,86 \cdot 0 \\
& =448 \cdot 5,35 \mathrm{I} \cdot 3,257 \cdot 8, \mathrm{I} 68 \cdot 9 \\
& =344 . \mathrm{I}, 289.2,2 \mathrm{I} 8.4 \text {, I } 44.9 \\
& =292 \cdot 3,227 \cdot 9,164 \cdot 9 \text {, 106.8 }
\end{aligned}
$$

Irregular terms :

$\begin{array}{llll}J=\frac{9}{2}, \frac{7}{2}, \frac{5}{2}, \frac{5}{2}, \frac{3}{2} & x, & =4 \mathrm{II} \cdot 2,294.4, \mathrm{I} 45.4, & 70 \cdot 2 \\ J=\frac{1}{2}, \frac{9}{2}, \frac{7}{2}, \frac{5}{2} & y, & =474 \cdot 9,354 \cdot 3,244.8\end{array}$

The twenty multiplets consist of the following combinations (numbering of F. M. Walters) :

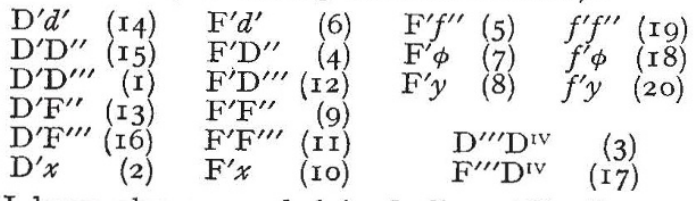

I have also succeeded in finding still other combinations of these terms, e.g. the multiplets $D^{\prime} y, \mathrm{D}^{\prime} \phi$, $f^{\prime} F^{\prime \prime}$.

The University, Leyden,

S. Goudsmit February I6.

NO. 2843, VO: . I I 3 ]
As the subject to which Mr. Goudsmit refers has recently been dealt with by other scientific workers, it is due to him to direct attention to the date of his letter. The delay in publication is due to the fact that the proof sent was not returned, and a duplicate afterwards posted reached us only on April I I.

ED. NATURe.

\section{The "Bleeding" of Cut Trees in Spring.}

IN the correspondence on the above subject in NATuRE of April 5, p. 492, Prof. Priestley refers to me by name, though he honours me unduly with the title of Dr., to which I can lay no claim. In a small way I have been engaged in tree-planting for the last twenty years, and often go among my saplings in winter with long pruners, cutting out double leaders and other ill-placed shoots. The profuse bleeding from cut surfaces of the sycamore and birch has struck me so forcibly that now, unless their pruning is completed by February, I refrain from dealing with them until foliation commences, imagining that the loss of so much saccharine sap must be detrimental. The bleeding has not been noticed in the case of other forest trees commonly grown in this country. No doubt there are other species of maple (Acer) and birch (Betula) that bleed readily on being injured in early spring. In fact, I feel fairly certain that the Norway Maple (Acer platanoides) does so, and of course the Sugar Maple of Canada is a stock example put to economic use. Recently I tried some young English Maples (Acer campestre), but they failed to respond; though a sycamore adjoining bled at once on being cut. It would be interesting to ascertain how far this spring bleeding is characteristic for the above two genera.

In Cumberland the spring so far has been abnormally dry-in fact, an official drought has been registered-and this, I think, is affecting the bleeding. On two occasions recently, sycamores growing on a dry bank have been compared with others situated in the damp hollow below; the former on being cut exuded no sap, while the latter bled immediately.

There may be something in Mr. C. W. Folkard's idea of sunshine stimulating the flow. I have a feeling that way myself, but no systematic observations to offer with which to support it. Prof. Priestley dismisses this idea with the suggestion that the sunshine will raise the soil temperature and so increase root activity. But if it can be shown that as a rule a sunny day following a dull one provokes bleeding which was not in evidence before, then surely this sunshine can scarcely have had time to warm appreciably the soil so as to augment root activity. The extra heat will, however, make itself felt at once on the shoot system.

It is to me not a matter of surprise to learn that the sugar in the exuded sap of the sycamore is sucrose and not hexose. The little we know regarding the rôle of sugars in the flowering plants (Angiosperms) suggests that the plant, when calling upon its carbohydrate reserves (starch, inulin, etc.), is in a hurry to get these transformed into cane sugar (sucrose) for circulatory purposes. Though no reserve carbohydrate (raffinose and gentianose are somewhat exceptions), when hydrolysed by enzyme, or acid, yields sucrose, yet, strange to say, in the living plant it is this sugar which accumulates and none of those (such as maltose, glucose, fructose, mannose) resulting from the hydrolysis. The same may apply to the photosynthesis of carbohydrate in the green leaf. The sugar actually first liberated may be sucrose, though a hexose phase may be passed through rapidly, without any appreciable accumulation of this class of sugar. Accepting the view that 